\title{
Neuroschistosomiasis Presenting as Conus Medullaris Lesion; A Case Report
}

\author{
Al-Hashel JY ${ }^{1,2}$, Ibrahim MA ${ }^{1}$, John JK ${ }^{1}$, Ismail II $^{* 1}$, and Abdelnabi EA ${ }^{3}$ \\ ${ }^{1}$ Department of Neurology, Ibn Sina Hospital, Kuwait \\ ${ }^{2}$ Department of Medicine, Faculty of Medicine, Health Sciences Centre, Kuwait University, Kuwait \\ ${ }^{3}$ Department of Radiology, Ibn Sina Hospital, Kuwait
}

*Corresponding author: Ismail II, Department of Neurology, Ibn Sina Hospital, Kuwait, E-mail: dr.ismail. ibrahim2012@Gmail.com

Citation: Al-Hashel JY, Ibrahim MA, John JK, Ismail II, Abdelnabi EA (2018) Neuroschistosomiasis Presenting as Conus Medullaris Lesion; A Case Report. J Neurol Neurol Disord 4(2): 204. doi: 10.15744/2454-4981.4.204

Received Date: February 9, 2018 Accepted Date: August 23, 2018 Published Date: August 27, 2018

\begin{abstract}
Schistosomiasis is one of the most widespread parasitic diseases in the world. Spinal Cord Schistosomiasis (SCS) usually presents acutely or subacutely and classified into three clinical forms: medullary, myelo-radicular and conus-cauda equina syndrome. Despite the fact that Kuwait is not an endemic country for Schistosomiasis, many expats residing in this country are from endemic areas. We report a case of a 29 years old Egyptian male with conus-cauda equina syndrome with classic radiological findings and good response to treatment.
\end{abstract}

Keywords: Schistosoma; Conus; Cauda; Myelopathy

\section{Introduction}

Schistosomiasis is one of the most widespread parasitic diseases in the world. The three main species of Schistosoma; Schistosoma Mansoni, Schistosoma Haematobium and Schistosoma Japonicum can affect the central nervous system [1].

Spinal Cord Schistosomiasis (SCS) usually presents acutely or subacutely and is classified into three clinical forms: (a) Medullary with a predominance of spinal cord involvement; (b) Myeloradicular, with mainly spinal cord and nerve root involvement; and (c) Conus-cauda equina syndrome, with predominantly conus or cauda equina involvement [2].

The pathogenesis of the disease remains unclear, but host inflammatory response to schistosomal eggs lying in the nervous tissue represents the chief determinant of spinal cord lesion [3]. Clinical involvement of the spinal cord can manifest weeks to several years after exposure. Although uncommon, this complication is not rare in endemic areas for schistosomiasis and several cases have been reported in Europe and USA [4].

The disease is characterized by a triad consisting of lumbar/lower limb pain, paraparesis and/or altered sensation in the lower limbs, and urinary dysfunction. The diagnosis is based upon: (i) the presentation of neurological symptoms and signs resulting from lesions of the spinal cord; (ii) the demonstration of schistosomal infection using microscopy and serological techniques, and (iii) the exclusion of other causes of myelopathy [1].

Treatment is based on a combination of antischistosomal drugs and corticosteroids [7,8].

\section{Case Summary}

A 29 year-old Egyptian male working in Kuwait for one year who was previously as a farmer in Egypt before coming to Kuwait. He was admitted to our hospital with burning sensation during micturition for 2 days followed by paraesthesia in the buttocks and upper thigh with retention of urine, constipation and erectile dysfunction. No history of cranial nerve, motor or cerebellar affection. The general physical examination was normal but neurological evaluation revealed mild proximal lower limb weakness with muscular strength $4+$ out of 5 according to the Medical Research Council grading. Deep tendon reflexes of lower limbs were brisk knee reflexes and bilateral absent ankle reflexes and Babinski sign was bilaterally present. Decreased pain and touch sensation in the saddle area. Decreased deep sensation i.e. vibratory and joint position sense, was also noticed in lower limbs. No cognitive, cranial nerve or upper limb disturbances were noted. Based on his symptoms and examination, localization was suggestive of conus and epiconus lesion. 
Blood cell counts showed mild increase in eosinophils. Blood biochemistry and chest X-ray were normal. Urine analysis showed increased RBCs and presence of Schistosoma Haematobium ova. Serological studies for HIV, human T lymphotropic virus (HTLV), hepatitis B, and C and syphilis were negative. Indirect immunofluorescence test for antibodies to S. Haematobium was positive for serum. Cerebrospinal fluid (CSF) revealed $1.5 \mathrm{gm} / \mathrm{dl}$ of protein, 22 white cells/mm ( $90 \%$ lymphocytes) and $30 \mathrm{mg} / \mathrm{dl}$ of glucose. There was no alteration on magnetic resonance imaging (MRI) of the brain and cervicodorsal spine.

MRI of the lumbosacral spine demonstrated increased signal intensity on T2-weighted sequences in the conus region with gadolinium enhancement (Figure 1). A diagnosis of SCS was made and the patient was treated with oral Praziquantel (60 mg/kg for 3 days) and Prednisolone $1 \mathrm{mg} / \mathrm{kg}$ for 1 month and gradual withdrawal over 6 months. On patient follow-up, he was markedly improved. A new MRI spinal study performed 3 months after treatment showed impressive improvement of the lesions.

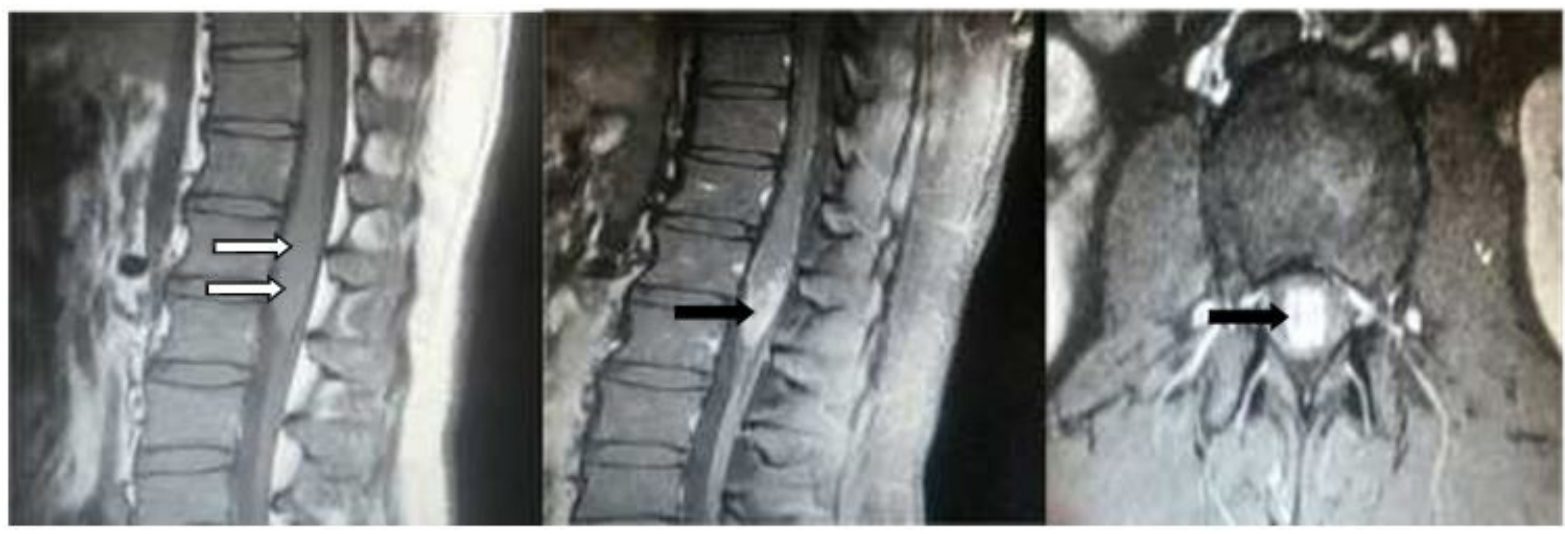

Figure 1: A. Sagittal T1-weighted image shows moderate expansion of the distal cord and conus medullaris (white arrows). B and C. Postcontrast, Sagittal and axial images, in which the lesion is well delineated by contrast enhancement (black arrows)

\section{Discussion}

Schistosomiasis is a helminthic infection caused by blood flukes of the genus Schistosoma. It is one of the most widespread parasitic diseases in the world, and is an important public health problem, particularly in tropical areas [9].

Neuroschistosomiasis refers to schistosomal involvement of the CNS. Almost all reported cases of Neuroschistosomiasis are caused by infection with Schistosoma Mansoni, Schistosoma Haematobium, or Schistosoma Japonicum. With symptomatic involvement of the CNS, S. Mansoni and S. Haematobium usually affects the spinal cord, whereas S. Japonicum causes mostly encephalitis [2].

The differential diagnosis of conus lesion comprises several conditions; infectious, inflammatory, vascular and demyelinating diseases. SCS, however, differs from other causes of myelitis in that it commonly affects the lower cord, whereas transverse myelitis commonly affects the midthoracic or cervical regions.

Although the clinical picture of SCS is non-specific, it should be strongly considered in patients aged 5-35 years from endemic areas who present with pain in the lower back or lower limbs before developing rapidly progressive weakness and sensory impairment in the lower limbs associated with autonomic disorders, especially bladder dysfunction. Clinical diagnosis becomes less likely when higher segments are affected or when the symptoms progress more slowly. The most suggestive manifestations are lower spinal cord involvement, acute or subacute onset, and involvement of both medulla and spinal roots, particularly the cauda equina roots. Rapid and pronounced improvement after treatment lends further support to diagnosis $[5,6]$.

The diagnosis of SCS in our case was based on clinical history of subacute neurological deficit, the endemicity of Schistosoma in Egypt, and laboratory and imaging results.

CSF examination usually shows slight-to-moderate increases in both total protein concentration and lymphocytic count. Eosinophils are present in the CSF in about $50 \%$ of patients $[5,10]$.

In our patient CSF analysis reflected an inflammatory pattern characterized by increase in the total protein concentration and pleocytosis. Eosinophils were not found. Although the presence of eosinophils in the CSF can contribute to the diagnosis of spinal schistosomiasis, it is reported only in $40 \%$ of patients and is nonspecific, because this cell can be seen in several other conditions.

MRI is very sensitive in the detection of abnormalities in patients with SCS, but the alterations are non-specific. The most common findings are signal hyperintensity on T2-weighted images, enlargement of the spinal cord (particularly lower cord and conus medullaris), thickening of the spinal roots (especially cauda equina roots), and a heterogeneous pattern of contrast enhancement on T1-weighted images [10-12].

In our case, MRI study showed a conus medullaris hyperintense lesion. Interestingly, the MRI pattern observed in our patient, i.e., 
hyperintense signal in T2-weighted sequences and heterogeneous enhancement following contrast injection, has been consistently associated with Schistosoma Mansoni.

A definite diagnosis of SCS would require histological examination of the spinal cord lesion. As this procedure has potential serious consequences, it has been used less frequently [13].

SCS treatment involves the use of schistosomicidal drugs and corticosteroids. Praziquantel is the treatment of choice for all Schistosoma species. Adverse effects are generally mild and include dizziness, headache, lassitude, abdominal pain, and pain in lower limbs. The doses most widely recommended are $40-60 \mathrm{mg} / \mathrm{kg}$ per day for 1 to 3 days [2].

According to most investigators, SCS should be treated with Praziquantel and a steroid, but the recommended doses vary between studies $[6,7,11]$. However, other reports have described successful treatment of several patients with Praziquantel, with or without a steroid $[15,16]$. About $65 \%$ of patients with SCS who are treated early recover completely or are left with negligible deficits that do not cause any functional limitations-the remaining patients are left with some sequelae that vary from mild to severe [2].

We have successfully treated our patients with $60 \mathrm{mg} / \mathrm{kg}$ a day of Praziquantel for 3 days instead of giving the drug for $1 \mathrm{day}$ only. We used this dosage over 3 days to ensure central penetration and effectiveness. Also, our patient received Prednisolone 1mg/ $\mathrm{kg}$ for 4 weeks which was tapered over 6 months. He had a complete neurological and radiological recovery following treatment.

\section{Conclusion}

Spinal Cord Schistosomiasis (SCS) must be considered in the differential diagnosis of myeloradiculopathies exhibited by patients from endemic areas. This is the first case report from Kuwait to be published in literature. Physicians working in areas where Schistosomiasis is not endemic should also consider the diagnosis of SCS when treating patients returning from countries of endemicity who have clinical signs and symptoms of myeloradiculopathy.

\section{References}

1. Lambertucci JR, Silva LC, do Amaral RS (2007) Guidelines for the diagnosis and treatment of schistosomal myeloradiculopathy. Rev Soc Bras Med Trop 40: 574-81.

2. Ferrari TC, Moreira PR (2011) Neuroschistosomiasis: clinical symptoms and pathogenesis. Lancet Neurol 10: 853-64.

3. Pittella JEH (1997) Neuroschistosomiasis. Brain Pathol 7: 649-62.

4. Gomes CM, Hisano M, Machado LR, Figueiredo JA, Lucon AM, et al. (2005) Urological manifestations of chronic schistosomal myeloradiculopathy. BJU Int 96: 853-6.

5. Ferrari TC, Moreira PR, Cunha AS (2004) Spinal cord schistosomiasis: a prospective study of 63 cases emphasizing clinical and therapeutic aspects. J Clin Neurosci 11: 246-53

6. Ferrari TCA, Moreira PRR, Cunha AS (2008) Clinical characterization of neuroschistosomiais due to Schistosoma mansoni and its treatment. Acta Trop 108: 89-97.

7. Santos EC, Campos GB, Diniz AC, Leal JC, Rocha MO (2001) Clinical profile and criteria for the diagnosis of schistosomotic myeloradiculopathy. Arq Neuropsiquiatr 59: 772-7.

8. Peregrino AJ, Puglia PM, Nobrega JP, Livramento JA, Marques-Dias MJ (2002) Schistosomiasis of the spinal cord: analysis of 80 cases. Arq Neuropsiquiatr 60 : 603-8.

9. Steinmann P, Keiser J, Bos R, Tanner M, Utzinger J (2006) Schistosomiasis and water resources development: systematic review, meta-analysis, and estimates of people at risk. Lancet Infect Dis 6: 411-25.

10. Silva LC, Maciel PE, Ribas JG, Souza-Pereira SR, Antunes CM, et al. (2004) Treatment of schistosomal myeloradiculopathy with praziquantel and corticosteroids and evaluation by magnetic resonance imaging: a longitudinal study. Clin Infec Dis 39: 1619-24.

11. Lambertucci JR, dos Santos Silva LC, Andrade LM, de Queiroz LC, Carvalho VT (2008) Imaging techniques in the evaluation of morbidity in Schistosomiasis mansoni. Acta Trop 108: 209-17.

12. Saleem S, Belal AI, El-Ghandour NM (2005) Spinal cord schistosomiasis: MR imaging appearance with surgical and pathologic correlation. AJNR Am J Neuroradiol 26: 1646-54.

13. Cohen-Gadol AA, Zikel OM, Miller GM, Aksamit AJ, Scheithauer BW, et al. (2003) soinal cord biopsy: a review of 38 cases. Neurosurgery J 52: 815-81.

14. Ferrari TC (1999) Spinal cord schistosomiasis: a report of 2 cases and review emphasizing clinical aspects. Medicine 78: 176-90.

15. Roberts M, Cross J, Pohl U (2006) Cerebral schistosomiasis. Lancet Infect Dis 6: 820.

16. Fowler R, Lee C, Keystone JS (1999) The role of corticosteroids in the treatment of cerebral schistosomiasis caused by Schistosoma mansoni: case report and discussion. Am J Trop Med Hyg 6: 47-50. 


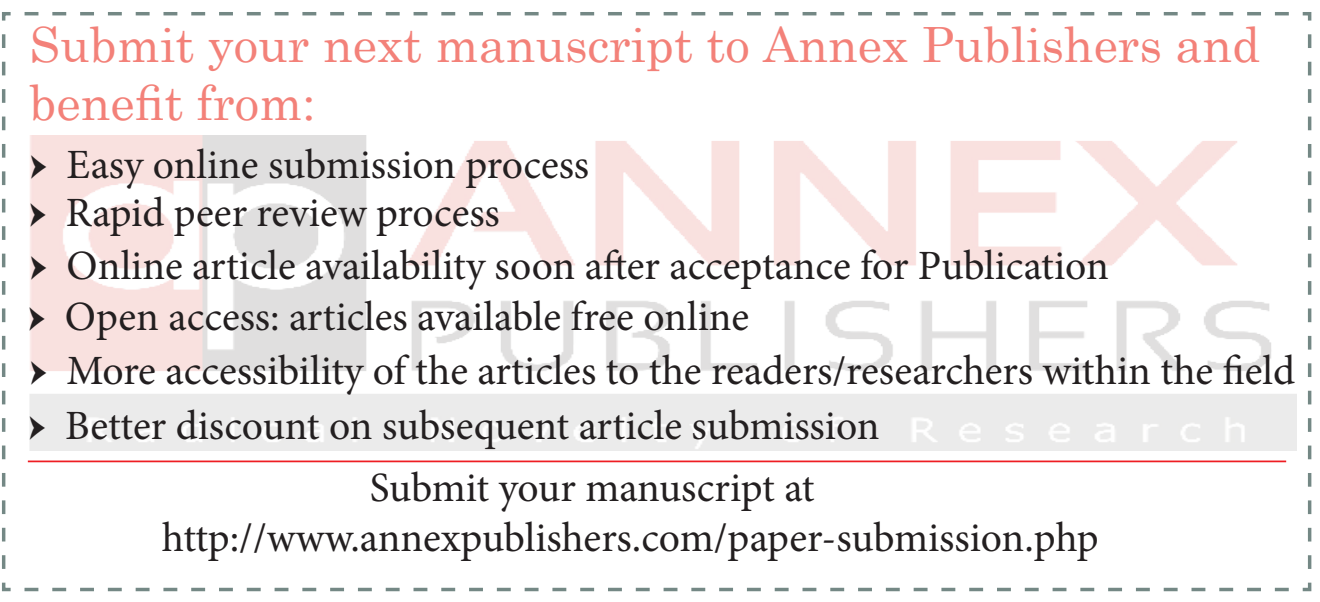

